\title{
pGBbBShift \\ Perceptual Generalized Bitplane-by-Bitplane Shift
}

\author{
Jaime Moreno \\ Superior School of Mechanical and Electrical Engineers, \\ National Polytechnic Institute of Mexico, \\ IPN Avenue, Lindavista, Mexico City, 07738, Mexico.
}

\begin{abstract}
The paper we present pGBbBShift. This algorithm permits to code any Region of Interest (ROI) in a perceptual way, i.e. the presented algorithm introduces some characteristics of the HumanVisual System. Furthermore, it introduces features of chromatic induction to the GBbBShift method when bitplanes of ROI and background areas are coded. Thus, the included features balance visual importance of some pixels regardless their numerical importance, namely we avoid to use Information Theory criteria. Visual criteria are applied using the CIWaM, which is contrast band-pass filter that predicts color perception. pGBbBShift is compared against classicalROI algorithms, such as the MaxShift method of JPEG2000 and results show that there is no perceptual difference. pGBbBShift method is an open algorithm that can be applied in any wavelet based image coder such as JPEG2000, SPIHT or SPECK. Finally, we applied pGBbBShift to Hi-SET coder and we obtain the best results when the overall visual image quality is assessed
\end{abstract}

Keywords-Image Coding; JPEG2000; Hi-SET; region of interest(ROI); bitplane coding; wavelet coding; maximum shift (MaxShift);bitplane-by-bitplane shift (BbBShift); generalized bitplane-by-bitplane shift (GBbBShift)

\section{INTRODUCTION}

\section{A. JPEG2000ROI Coding}

Region of interest (ROI) image coding is a feature that modern image coders have, which allows to encode an specific region with better quality than the rest of the image or background (BG). ROI coding is one of the requirements in the JPEG2000 image coding standard [1], [2], which defines twoROI methods[3], [4]:

1) Based on general scaling [3]

2) Maximum shift(MaxShift)[4]

The general ROI scaling-based method scales coefficients in such a way that the bits associated with the ROI are shifted to higher bitplanes than the bitplanes associated with the background, as shown in Figure 1(b). It implies that during a embedded coding process, any background bitplane of the image is located after the most significant ROI bitplanes into the bitstream. But, in some cases, depending on the scaling value, $\varphi$, some bits of ROI are simultaneously encoded with BG. Therefore, this method allows to decode and refine the ROI before the rest of the image. No matter $\varphi$, it is posible to reconstruct with the entire bitstream a highest fidelity versionof the whole image. Nevertheless, If the bitstream is terminated abruptly, theROI will havea higher fidelity than BG.

The scaling-based method is implemented in five steps:.
1) A wavelet transform of the original images is performed.

2) AROI mask is defined, indicating the set of coefficients that are necessary for reaching a lossless.

3) Wavelet coefficients are quantized and stored in a sign magnitude representation, using the most significant part of the precision. It will allow to downscale BG coefficients.

4) A specified scaling value, $\varphi$ ', downscales the coefficientsinside the $B G$.

5) The most significant bitplanes are progressively entropyencoded.

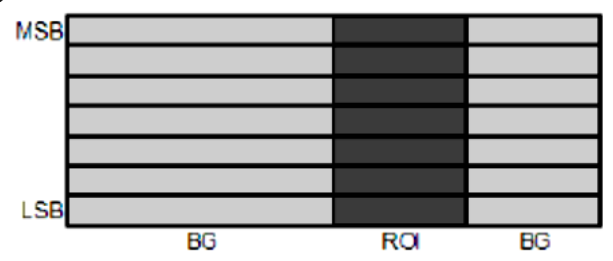

(a) No ROI coding

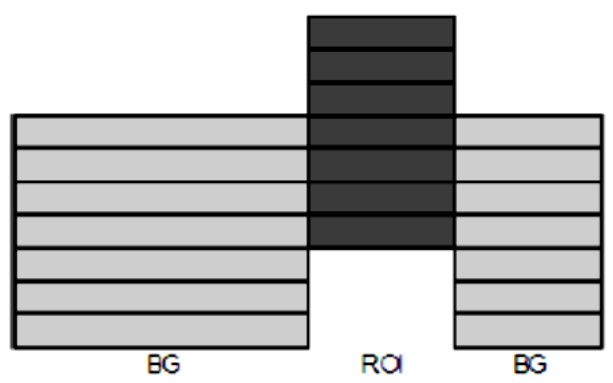

(b) Scaling Based Method

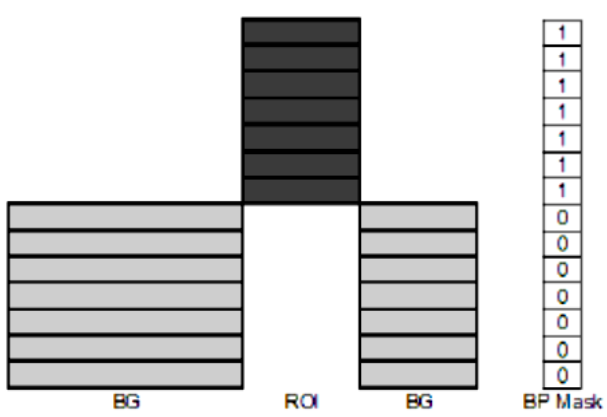

(c) MaxShift method

Fig. 1. JPEG2000 ROI Coding. (a) No ROI coding, (b) Scaling based ROI coding method $(\varphi=3)$ and (c) MaxShift method, $\varphi=7$. Background is denoted as BG, Region of Interest as ROI and Bitplane mask as $B P_{\text {mask }}$. MSB is the most significant bitplane and LSB is the least significant bitplane. 

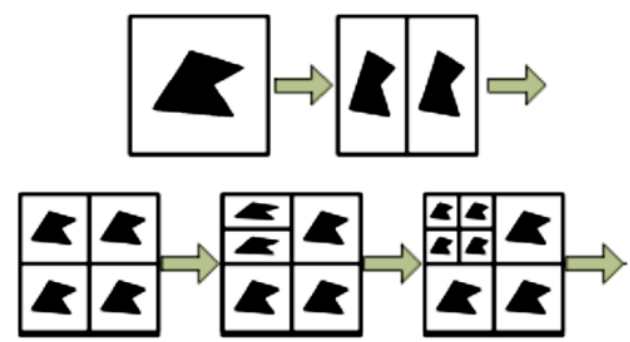

Fig. 2. ROI mask generation, wavelet domain.

The inputofROI scaling-based methodis the scalingvalue $\varphi$, while MaxShift method calculates it. Hence, the encoder defines from quantized coefficients this scaling value such that:

$$
\varphi=\left\lceil\log _{2}\left(\max \left\{\mathcal{M}_{\mathcal{B G}}\right\}+1\right)\right\rceil
$$

where $\max \left\{\mathcal{M}_{\mathcal{B C}}\right\}$ is the maximum coefficient in the BG. Thus, when ROI is scaled up $\varphi$ bitplanes, the minimum coefficient belonging to ROI will be place one bitplane up of BG (Fig. 1(c)). Namely, $2^{\varphi}$ is the smallest integer that is greater than any coefficient in the BG. MaxShift method is shown in Figure 1(c). Bitplane mask (BPmask) will be explained in section II-B.

At the decoder side, the ROI and BG coefficients aresimply identified by checking the coefficient magnitudes. Allcoefficients that are higher or equal than the 甲thbitplanebelong to the ROI otherwise they are a part of BG. Hence, itis not important to transmit the shape information of the ROIor ROIs to the decoder. The ROI coefficients are scaled down $\varphi$ bitplanes before inverse wavelet transformation is applied.

\section{B. Perceptual Coding}

1) Chromatic Induction Wavelet Model: In order to generate an approximation to how every pixel is perceived from a certain distance taking into account the value of its neighboring pixels the Chromatic Induction Wavelet Model (CIWaM) is used. CIWaM attenuates the details that the human visual system is not able to perceive, enhances those that are perceptually relevant and produces an approximation of the image that the brain visual cortex perceives. CIWaM takes an input image I and decomposes it into a set of wavelet planes $\omega_{s, o}$ of different spatial scales $s$ (i.e., spatial frequency v) and spatial orientations $o$. It is described as:

$$
\mathcal{I}=\sum_{s=1}^{n} \sum_{o=v, h, d g l} \omega_{s, o}+c_{n},
$$

where $\mathrm{n}$ is the number of wavelet planes, $\mathrm{c}_{\mathrm{n}}$ is the residualplane and $\mathrm{o}$ is the spatial orientation either vertical, horizontalor diagonal. The perceptual image $I_{\rho}$ is recovered by weightingthese $\omega_{\mathrm{s}, \mathrm{o}}$ wavelet coefficients using the extended ContrastSensitivity Function (e-CSF), which considers spatial surround information (denoted by $r$ ), visual frequency ( $v$ related tospatial frequency by observation distance) and observationdistance (d). Perceptual image $\mathrm{I}_{\rho}$ can be obtained by

$$
\mathcal{I}_{\rho}=\sum_{s=1}^{n} \sum_{o=v, h, d g l} \alpha(\nu, r) \omega_{s, o}+c_{n},
$$

where $\alpha(v, r)$ is the e-CSF weighting function that tries toreproduce some perceptual properties of the HVS. The term $\alpha(v, \quad r) \quad \omega_{\mathrm{s}, \mathrm{o}} \equiv \omega_{\mathrm{s}, \mathrm{o} ;} \rho_{\text {,d }}$ can be considered the perceptualwavelet coefficients of image I when observed at distance d.For details on the CIWaM and the $\alpha(v, r)$ function, see [5].

2) Quantization: We employ the perceptual quantizer $(\rho S Q)$ either forward (F- $\rho S Q)$ and inverse (I- $\rho S Q$. Quantization is the only causethat introduces distortion into a compression process. Eachtransform sample at the perceptual image I $\rho$ (from Eq. 3) ismapped independently to a corresponding step size either $\Delta$ sor $\Delta n$, thus I $\rho$ is associated with a specific interval on the realline. Then, the perceptually quantized coefficients $Q$ ( $F-\rho S Q)$,from a known viewing distance $d$, are calculated as follows:

$\mathcal{Q}=\sum_{s=1}^{n} \sum_{o=v, h, d} \operatorname{sign}\left(\omega_{s, o}\right)\left\lfloor\frac{\left|\alpha(\nu, r) \cdot \omega_{s, o}\right|}{\Delta_{s}}\right\rfloor+\left\lfloor\frac{c_{n}}{\Delta_{n}}\right\rfloor$

The perceptual inverse quantizer (I- $\rho S Q)$ or the recovered $\alpha^{\prime}(v, r)$ introduces perceptual criteria to the classical InverseScalar Quantizer and is given by:

$$
\widehat{\mathcal{I}}= \begin{cases}\sum_{s=1}^{n} \sum_{o=v, h, d} \operatorname{sign}\left(\widehat{\omega_{s, o}}\right) \frac{\Delta_{s} \cdot\left(\left|\widehat{\omega_{s}^{o}}\right|+\delta\right)}{\widehat{\alpha}(\nu, r)} & \\ +\left(\left|\widehat{c_{n}}\right|+\delta\right) \cdot \Delta_{n}, & \left|\widehat{\omega_{s, o}}\right|>0 \\ 0, & \widehat{\omega_{s, o}}=0\end{cases}
$$

\section{RELATED WORK}

\section{A. BbBShift}

Wang and Bovik proposed the bitplane-by-bitplane shift(BbBShift) method in [6]. BbBShift shifts bitplanes on abitplane-by-bitplane strategy. Figure 3(a) shows an illustrationof the BbBShift method. BbBShift uses two parameters, $\varphi_{1}$ and $\varphi_{2}$, whose sum is equal to the number of bitplanes forrepresenting any coefficient inside the image, indexing thetop bitplane as bitplane 1. Summarizing, the BbBShift methodencodes the first $\varphi 1$ bitplanes with ROI coefficients, then, BGand ROI bitplanes are alternately shifted, refining graduallyboth ROI and BG of the image (Fig. 3(a)). The encodingprocess of the BbBShift method is defined as:

1) For a given bitplane bpl with at least one ROI coefficient:

- If $b p l \leq \varphi_{1}, b p l$ is not shifted.

- If $\varphi_{1}<b p l \leq \varphi_{1}+\varphi_{2}, b p l$ is shifted down to $\varphi_{1}+2\left(b p l-\varphi_{1}\right)$

2) For a given bitplane bpl with at least one $B G$ coefficient:

- If $b p l \leq \varphi_{2}, b p l$ is shifted down to $\varphi_{1}+2 b p l-1$

- If $b p l>\varphi_{2}, b p l$ is shifted down to $\varphi_{1}+\varphi_{2}+b p l$ 


\section{B. GBbBShift}

In practice, the quality refinement pattern of the ROI andBG used by BbBShift method is similar to the general scalingbased method. Thus, when the image is encoded and thisprocess is truncated in a specific point the quality of the ROIis high while there is no information of BG.
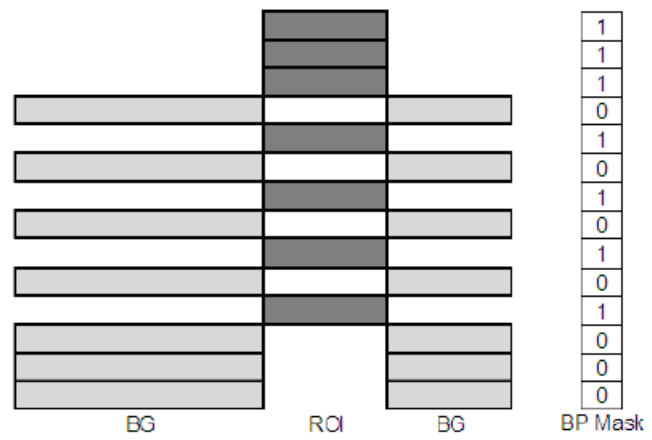

(a) BbBShift

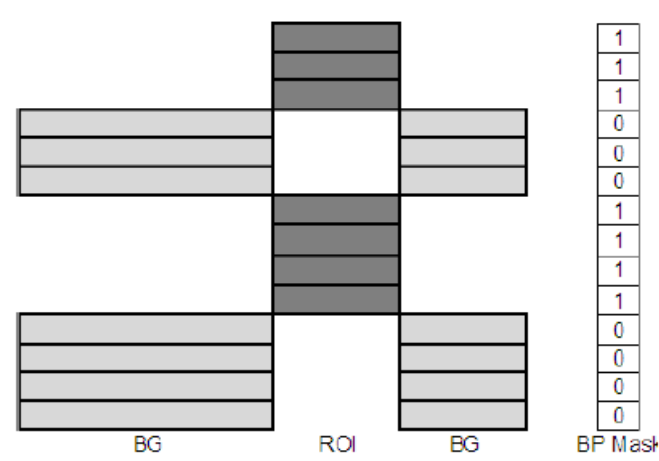

(b) GBbBShift

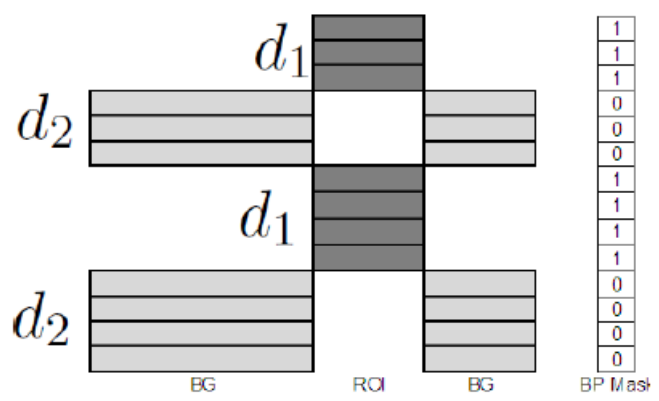

(c) $\rho$ GBbBShift

Fig. 3. ROI Coding Methods. (a) BbBShift, $\varphi_{1}=3$ and $\varphi_{2}=4$, (b) GBbBShift and (c) $\rho$ GBbBShift. Background is denoted as BG (For $\rho$ GBbBShift method is perceptually quantized by $\rho \mathrm{SQ}$ at $d_{2}$ ), Region of Interest as ROI (For $\rho$ GBbBShift method is perceptually quantized at $d_{1}$ by $\rho \mathrm{SQ})$ and Bitplane mask as $B P_{\text {mask }}$.

Hence, Wang and Bovik [7] modified BbBShift method andproposed the generalized bitplane-by-bitplane shift (GBbBShift)method, which introduces the option to improve visualquality either of ROI or BG or both. Figure 3(b) shows thatwith GBbBShift method it is posible to decode some bitplanesof BG after the decoding of same ROI bitplanes. It allowsto improve the overall quality of the recovered image. Thisis posible gathering BG bitplanes.
Thus, when the encoding process achieves the lowest bitplanes of ROI, the quality of BGcould be good enough in order to portray an approximationof BG.

Therefore, the main feature of GBbBShift is to give theopportunity to arbitrary chose the order of bitplane decoding,grouping them in ROI bitplanes and BG bitplanes. This isposible using a binary bitplane mask or BPmask, whichcontains one bit per each bitplane, that is, twice the amount ofbitplanes of the original image. A ROI bitplane is representedby 1 , while a $\mathrm{BG}$ bitplane by 0 .

For example, the BPmask forMaxShift method in Figure 1(c) is 11111110000000, whilefor BbBShift in Figure 3(a) and GBbBShift in Figure 3(b) are11101010101000 and 11100011110000 , respectively.

At the encoder side, the BPmask has the order of shiftingboth the ROI and BG bitplanes. Furthermore, BPmask isencoded in the bitstream, while the scaling values $\varphi$ or $\varphi_{1}$ and $\varphi_{2}$ from the MaxShift and BbBShift methods, respectively, have to be transmitted.

\section{PGBBBSHIFT METHOD}

In order to have several kinds of options for bitplane scalingtechniques, a perceptual generalized bitplane-bybitplaneshift( $\rho$ GBbBShift) method is proposed. The $\rho$ GBbBShiftmethod introduces to the GBbBShift method perceptual criteriawhen bitplanes of ROI and BG areas are shifted.

This additionalfeature is intended for balancing perceptual importanceof some coefficients regardless their numerical importance andfor not observing visual difference at ROI regarding MaxShiftmethod, improving perceptual quality of the entire image.

Thus, $\rho$ GBbBShift uses a binary bitplane mask or BPmaskin the same way that GBbBShift (Figure 3(c)). At the encoder,shifting scheme is as follows:

1) Calculate $\varphi$ using Equation 1 .

2) Verify that the length of $B P_{\text {mask }}$ is equal to $2 \varphi$.

3) - For all ROI Coefficients, forward perceptual quantize them using Equation 4 (F- $\rho \mathrm{SQ})$ with viewing distance $d_{1}$.

- For all BG Coefficients, forward perceptual quantize them using Equation 4 (F- $\rho \mathrm{SQ})$ with viewing distance $d_{2}$, being $d_{2} \gg d_{1}$.

4) Let $\tau$ and $\eta$ be equal to 0 .

5) For every element $i$ of $B P_{\text {mask }}$, starting with the least significant bit:

- If $B P_{\text {mask }}(i)=1$, Shift up all ROI perceptual quantized coefficients of the $(\varphi-\eta)$-th bitplane by $\tau$ bitplanes and increment $\eta$.

- Else: Shift up all BG perceptual quantized coefficients of the $(\varphi-\tau)$-th bitplane by $\eta$ bitplanes and increment $\tau$.

At the decoder, shifting scheme is as follows: 
1) Let $\varphi=\frac{\text { length of } \bar{B} P_{\text {mask }}}{2}$ be calculated.

2) Let $\tau$ and $\eta$ be equal to 0 .

3) For every element $i$ of $B P_{\text {mask }}$, starting with the least significant bit:

- If $B P_{\text {mask }}(i)=1$, Shift down all perceptual quantized coefficients by $\tau$ bitplanes, which pertain to the

$(2 \varphi-(\tau+\eta))$-th bitplane of the recovered image and increment $\eta$.

- Else: Shift down all perceptual quantized coefficients by $\eta$ bitplanes, which pertain to the $(2 \varphi-(\tau+\eta))$-th bitplane of the recovered image and increment $\tau$.

4) Let us denote as $c_{i, j}$ a given non-zero wavelet coefficient of the recovered image with $2 \varphi$ bitplanes and $\bar{c}_{i, j}$ as a shifted down $c$ obtained in the previous step, with $\varphi$ bitplanes.

- If $\left(c_{i, j} \& B P_{\text {mask }}\right)>0$, inverse perceptual quantize $\bar{c}_{i, j}$ using Equation 5 (I- $\left.\rho \mathrm{SQ}\right)$ with $d_{1}$ as viewing distance.

- If $\left(c_{i, j} \& B P_{\text {mask }}\right)=0$, inverse perceptual quantize $\bar{c}_{i, j}$ using Equation 5 (I- $\left.\rho \mathrm{SQ}\right)$ with $d_{2}$ as viewing distance.

\section{EXPERIMENTAL RESULTS}

The $\rho$ GBbBShift method, as the other methods presentedhere, can be applied to many image compression algorithmssuch as JPEG2000 or Hi-SET. We test our methodapplying it to Hi-SET and the results are contrasted withMaxShift method in JPEG2000 and Hi-SET. The setupparameters are $\varphi=8$ for MaxShift and BPmask $=1111000110110000, \mathrm{~d} 1=5 \mathrm{H}$ and $\mathrm{d} 2=50 \mathrm{H}$, where $\mathrm{H}$ ispicture height (512 pixels) in a 19-inch LCD monitor, for $\rho \mathrm{GBbBShift}$. Also, we use the JJ2000 implementation whenan image is compressed by JPEG2000 standard[8].

\section{A. Application in well-known Test Images}

Figure 4 shows a comparison among methods MaxShift andGBbBShift applied to JPEG2000, in addition to, $\rho$ GBbBShiftapplied to Hi-SET. The 24-bpp image Barbara is compressedat 0.5 bpp. It can be observed that without visual differenceat ROI, the $\rho \mathrm{GBbBShift}$ method provide better imagequality at the BG than the general based methods defined inJPEG2000 Part II[1].

In order to better qualify the performanceof MaxShift, GBbBShift and $\rho$ GBbBShift methods, first,we compared these methods applied to the Hi-SET coderand then, we compare MaxShift and $\rho$ GBbBShift methodsapplied to the JPEG2000 standard and Hi-SET, respectively.

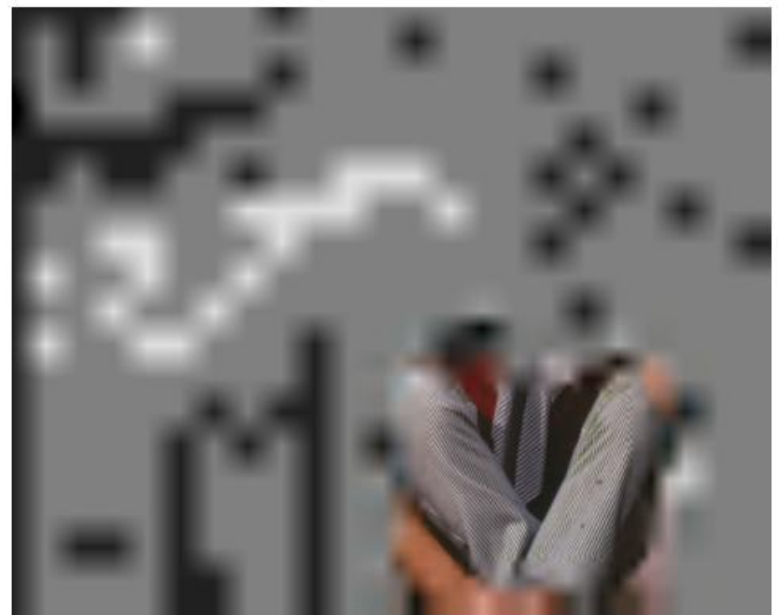

(a) MaxShift in JPEG2000 coder, 0.5 bpp

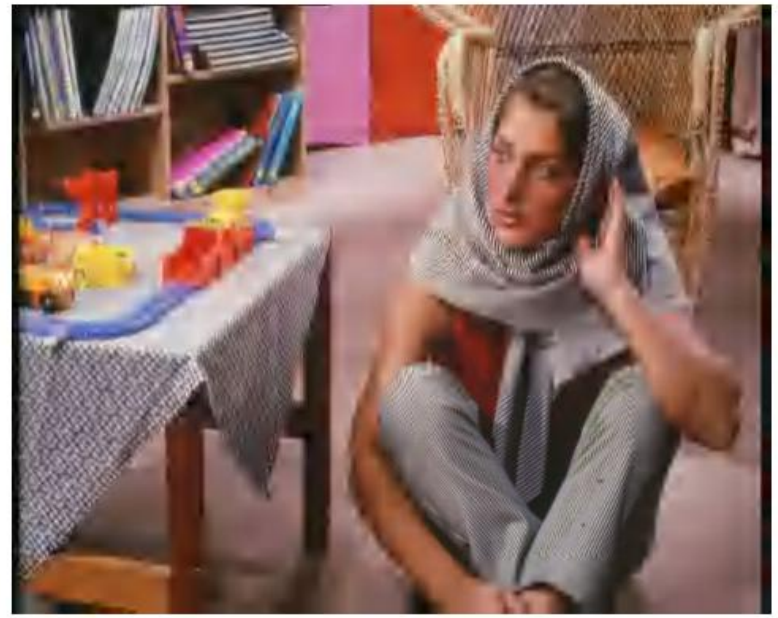

(b) GBbBShift in JPEG2000 coder, 0.5 bpp

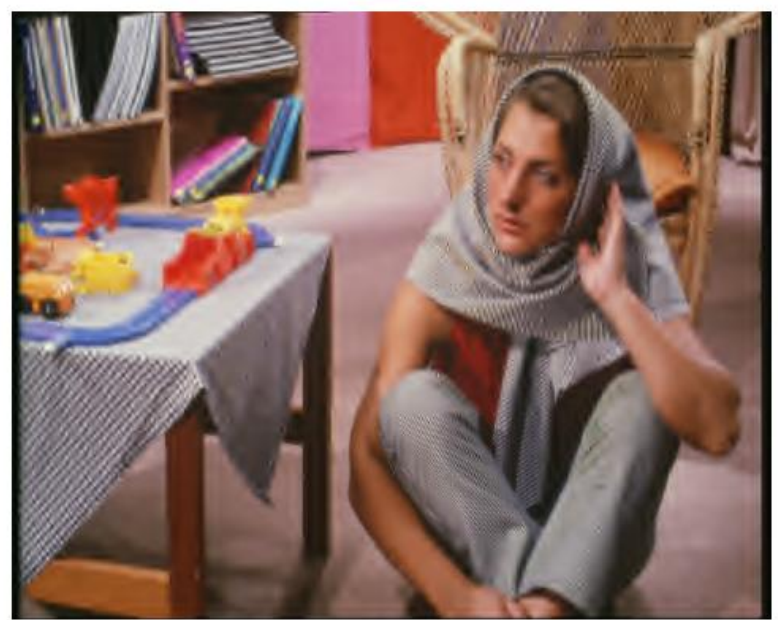

(c) $\rho$ GBbBShift in Hi-SET coder, 0.5 bpp

Fig. 4. $512 \times 640$ pixel Image Barbara with 24 bits per pixel. ROI is a patch of the image located at [341 280442442$]$, whose size is $1 / 16$ of the image. Decoded images at 0.5 bpp using MaxShift method in JPEG2000 coder((a) $\varphi=8)$, GBbBShift method in JPEG2000 coder ((b) $\left.B P_{\text {mask }}=1111000110110000\right)$ and $\rho \mathrm{GBbBSh}$ ift method in H $i$-SET coder $\left((\mathrm{c}) B P_{\text {mask }}=1111000110110000\right)$. 
We compress two different gray-scale and color images ofLenna at different bit-rates. ROI area is a patch at the centerof these images, whose size is $1 / 16$ of the image. We employthe perceptual quality assessment CwPSNR, which weights the mainstream PSNR by meansof a chromatic induction model.

Figs. 5(a) and 5(b) show the comparison among MaxShift(Blue Function), GBbBShift(Green Function) and $\rho$ GBbBShift(Red Function) methods applied to Hi-SET coder. $512 \times 512$ pixel Image Lenna for gray-scale is employ for this experiment. These Figures also show that the $\rho$ GBbBShift method gets the better results both in PSNR(objective image quality, Fig. 5(a)) and CwPSNR (subjective image quality, Fig. 5(b)) in contrast to MaxShift and GBbBShift methods. In addition, when MaxShift method applied to JPEG2000 coder and $\rho$ GBbBShift applied to Hi-SET coder are compared, $\rho$ GBbBShift obtains less objective quality (Fig. 5(c)), but better subjective quality for gray-scale images (Fig. 5(d)).

Figure 6 shows a visual example, when image Lenna is compressed at 0.34 bpp by JPEG2000 and Hi-SET. Thus, it can be observed that $\rho$ GBbBShift provides an important perceptual difference regarding the MaxShiftmethod(Fig. 6(d)). Furthermore, Figs. 6(b) and 6(c) show the examples when MaxShift and GBbBShift methods, respectively, are applied to the Hi-SET coder.

\section{B. Application in other image compression fields}

The usage of ROI coded images depends on an specificapplication, but in some fields such as manipulation andtransmission of images is important to enhance the imagequality of some areas and to reduce it in others[9], [10]. InTelemedicine or in Remote Sensing (RS) it is desirable tomaintain the best quality of the ROI area, preserving relevantinformation of $\mathrm{BG}$, namely the most perceptual frequencies.

Figure 7 shows an example of the application of ROI inRemote Sensing. Image 2.1.05, from Volumen 2: aerials ofUSC-SIPI image database 8 bits per pixel[11], is compressedat 0.42 bpp. MaxShift method spends all the bitratio forcoding ROI, located at [159 260384 460], while $\rho$ GBbBShiftbalances a perceptually lossless ROI area with an acceptablerepresentation of the BG.

Hence, the overall image qualitymeasured by PSNR in Figure 7(a) is $16.06 \mathrm{~dB}$, while inFigure $7(\mathrm{~b})$ is $24.28 \mathrm{~dB}$. When perceptual metrics assess theimage quality of the $\rho$ GBbBShift coded image, for example, VIFP $=0.4982$, WSNR $=24.8469$ and CwPSNR=27.07, whilefor MaxShift coded image $\mathrm{VIFP}=0.2368$, WSNR=11.33 andCwPSNR=16.72.

Thus, for this example, both PSNR and thesesubjective metrics reflect important perceptual differences betweenROI methods, being $\rho$ GBbBShift method better thanMaxShift method.

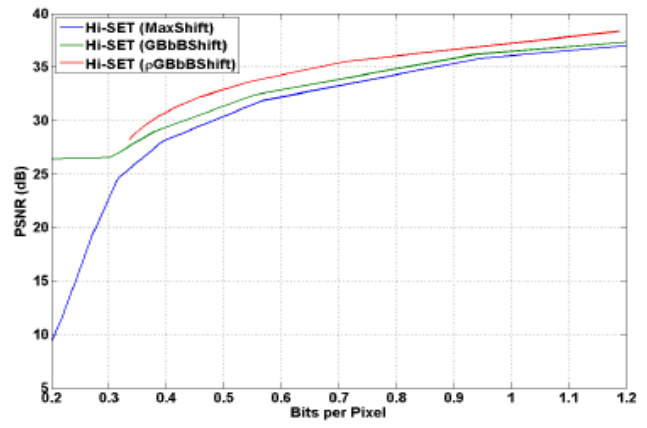

(a)

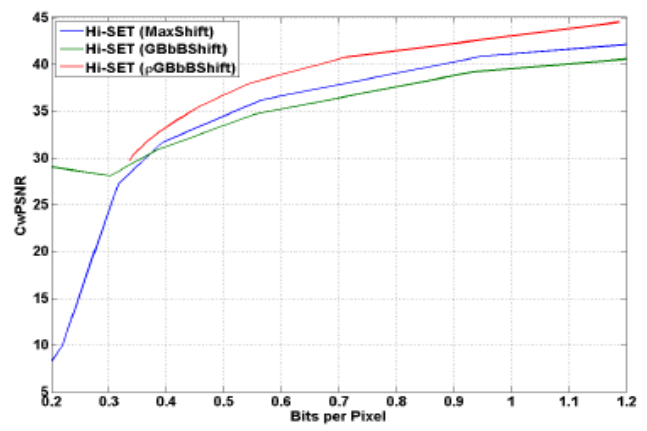

(b)

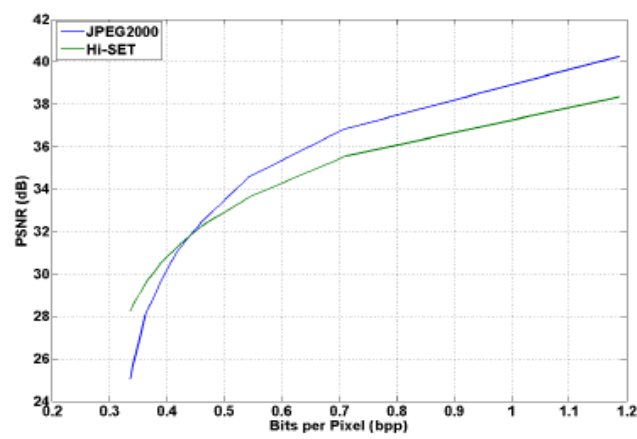

(c)

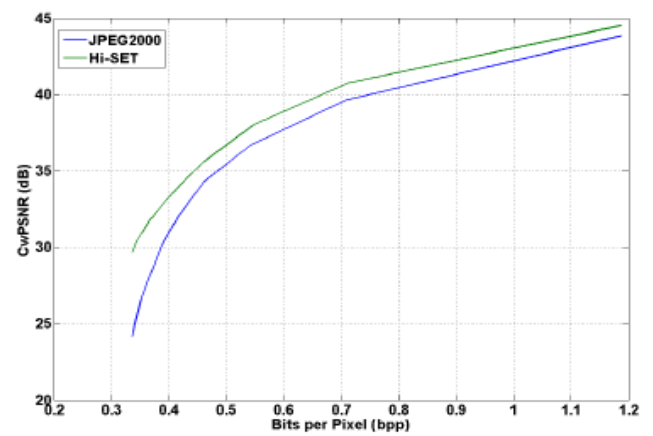

(d)

Fig. 5. (a-b) Comparison among MaxShift(Blue Function), GBbBShift(Green Function) and $\rho$ GBbBShift(Red Function) methods applied to $\mathrm{H} i$-SET coder. (c-d) Comparison between MaxShift method applied to JPEG2000 coder and $\rho$ GBbBShift applied to H $i$-SET coder. $512 \times 512$ pixel Image Lenna with 8 bits per pixel is employed for this experiment. ROI is a patch at the center of the image, whose size is $1 / 16$ of the image. The overall image quality of decoded images at different bits per pixel are contrasted both (a and c) objectively and (b and d)subjectively. 


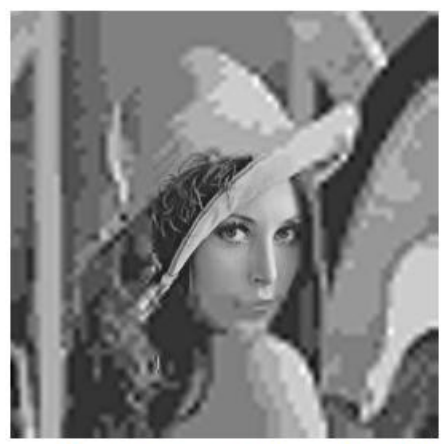

(a) MaxShift method in JPEG2000 coder, 0.34 bpp.

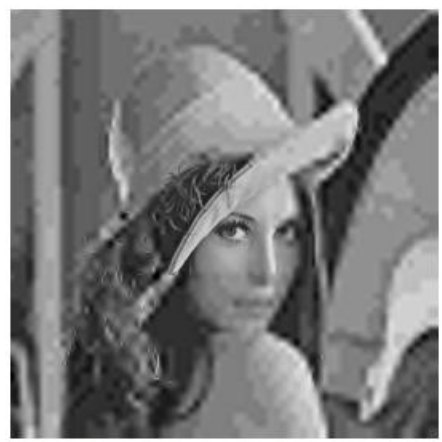

(b) MaxShift method in $\mathrm{Hi}$-SET coder, 0.34 bpp.

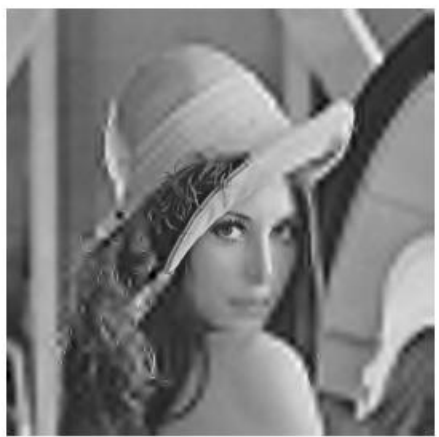

(c) GBbBShift method in $\mathrm{H} i$-SET coder, 0.34 bpp.

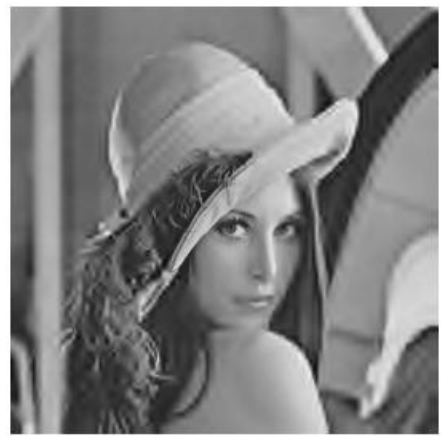

(d) $\rho$ GBbBShift method in $\mathrm{H} i$-SET coder, 0.34 bpp

Fig. 6. $512 \times 512$ pixel Image Lenna from CMU image database with 8 bits per pixel. ROI is a patch at the center of the image, whose size is $1 / 16$ of the image. Decoded images at 0.34 bpp using $\varphi=8$ for MaxShift method (a) in JPEG2000 coder and (b) in Hi-SET coder, and $B P_{\text {mask }}=$ 1111000110110000 for (c) GBbBShift and (d) $\rho$ GBbBShift methods in Hi-SET coder

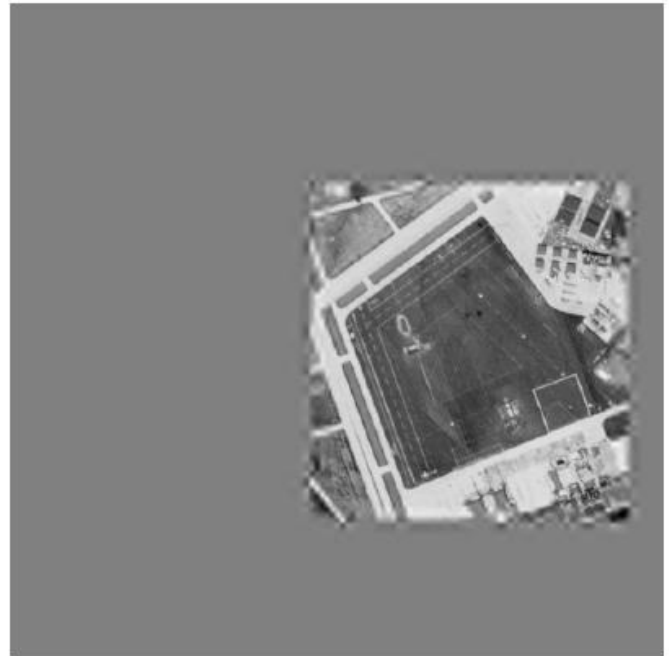

(a) MaxShift in JPEG2000 coder, 0.42 bpp

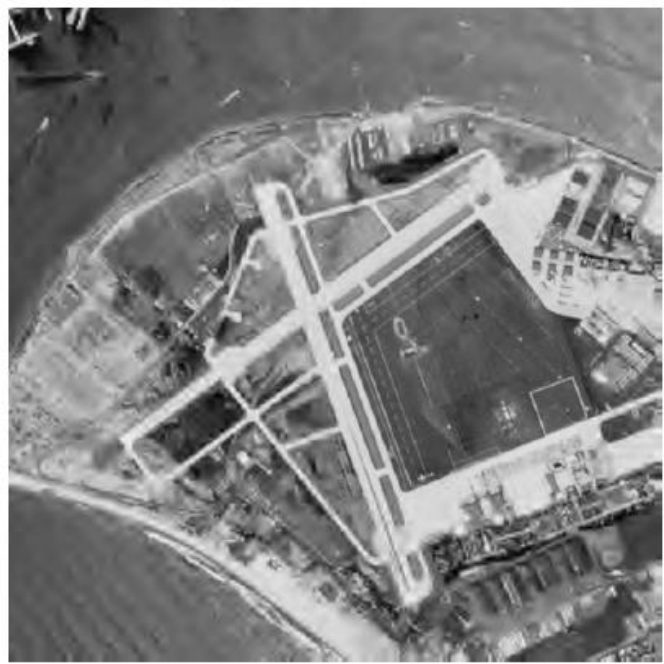

(b) $\rho$ GBbBShift method in Hi-SET coder, 0.42 bpp

Fig. 7. Example of a remote sensing application. $512 \times 512$ pixel Image 2.1.05 from Volumen 2: aerials of USC-SIPI image database at 8 bits per pixel. ROI is a patch with coordinates [159 260384 460], whose size is $225 \times 200$ pixels. Decoded images at 0.42 bpp using MaxShift methoc (a) $\varphi=8)$ in JPEG2000 coder and $\rho$ GBbBShift method ((b) $B P_{\text {mask }}=$ 1111000110110000) in Hi-SET coder.

\section{CONCLUSIONS}

A perceptual implementation of the Region of Interest, $\rho$ GBbBShift(), is proposed, which is a generalized methodthat can be applied to any wavelet-based compressor. Weintroduced $\rho$ GBbBShift method to the Hi-SET coder and itvisually improves the results obtained by previous methodslike MaxShift and GBbBShift.

Our experiments show that $\rho$ GBbBShift into Hi-SET provides an important perceptualdifference regarding the MaxShift method into JPEG2000, when it is applied to conventional images like Lenna orBarbara.

\section{ACKNOWLEDGMENT}

This work is supported by The National Polytechnic Institute of Mexico by means of a granted fund by the 
Committee of Operation and Promotion of Academic Activities (COFAA), in addition to the Project No. 20131312 granted by the Academic Secretary and National Council of Science and Technology of Mexico by means of Project No. 204151/2013.

\section{REFERENCES}

[1] M. Boliek, E. Majani, J. S. Houchin, J. Kasner, and M. Carlander, Information Technology: JPEG2000 Image Coding System (Extensions), JPEG 2000 Part II final committee draft ed., ISO/IEC JTC 1/SC 29/WG 1, Dec. 2000.

[2] M. Boliek, C. Christopoulos, and E. Majani, Information Technology: JPEG2000 Image Coding System, JPEG 2000 Part I final committee draft version 1.0 ed., ISO/IEC JTC1/SC29 WG1, JPEG 2000, April 2000.

[3] D. S. Taubman and M. W. Marcellin, JPEG2000: Image Compression Fundamentals, Standards and Practice, ser. ISBN: 0-7923-7519-X. Kluwer Academic Publishers, 2002.

[4] E. Atsumi and N. Farvardin, "Lossy/lossless region-of-interest image coding based on set partitioning in hierarchical trees," in International Conference on Image Processing, vol. 1, oct 1998, pp. 87 -91 vol.1. [5] X. Otazu, C. Párraga, and M. Vanrell, "Toward a unified chromatic induction model," Journal of Vision, vol. 10(12), no. 6, 2010.

[5] Z. Wang and A. C.Bovik, "Bitplane-by-bitplane shift ( BbBShift) - a suggestion for JPEG2000 region of interest image coding," IEEE Signal Processing Letters, vol. 9, no. 5, pp. 160 - 162, May 2002.
[6] Z. Wang, S. Banerjee, B. L. Evans, and A. C. Bovik, "Generalized bitplane-by-bitplane shift method for JPEG2000 ROI coding," IEEE International Conference on Image Processing, vol. 3, pp. 81-84, September 22-25 2002.

[7] C. Research, ' EcolePolytechniqueF'ed'erale de Lausanne, and Ericsson. (2001) JJ2000 implementation in Java, available at http://jj2000.epfl.ch/. annon Research, ' EcolePolytechniqueF'ed'erale de Lausanne and Ericsson. [Online]. Available: http://jj2000.epfl.ch/

[8] J. Bartrina-Rapesta, F. Auli-Llinas, J. Serra-Sagrista, A. Zabala-Torres, X. Pons-Fernandez, and J. Maso-Pau, "Region of interest coding applied to map overlapping in geographic information systems," in IEEE International Geoscience and Remote Sensing Symposium, 23-28 2007, pp. $5001-5004$.

[9] J. Gonzalez-Conejero, J. Serra-Sagrista, C. Rubies-Feijoo, and L. Donoso-Bach, "Encoding of images containing no-data regions withinJPEG2000 framework," in 15th IEEE International Conference on ImageProcessing, 12-15 2008, pp. $1057-1060$.

[10] S. and Image Processing Institute of the University ofSouthern California. (1997) The USC-SIPI image database, availableat http://sipi.usc.edu/database/. Signal and Image Processing Instituteof the University of Southern California. [Online]. Available:http://sipi.usc.edu/database/ 